\title{
CMEs and Flux Appearance in the Periphery of Two Unipolar Sunspots
}

\author{
X.L. Yang $\dagger$ W.B. Song, G.P. Zhou, J. Zhang, and J.X. Wang \\ National Astronomy Observatories, Chinese Academy of Sciences, Beijing 100012, China \\ email: yxl@ourstar.bao.ac.cn
}

\begin{abstract}
A class of large-scale magnetic compositions have been identified to be CME-prolific, which is characterized by a huge unipolar sunspot appearing in a large-scale extended bipolar region in synoptic magnetic charts. To understand the CMEs' origin and the nature of flux appearance, we scrutinize the long time-sequence of MDI magnetograms of high-resolution mode for super active region AR9236. Two types of magnetic features are clearly identified. They are moving magnetic features (MMFs) emanated radially from the penumbral boundary and emerging flux regions (EFRs) whose growing opposite polarities rotate out from the inner boundary of sunspot moat along helical paths in opposite directions. The interaction between the MMFs and EFRs often creates multi-fold magnetic neutral lines where the flare/CMEs initiated.
\end{abstract}

Keywords. CMEs,moving magnetic feature,emerging flux region

\section{Introduction}

A huge monopolar sunspot, which located in a favorite large-scale magnetic configuration and presented persistent flux appearance, is often flare/CMEs creative. For example, in NOAA AR9236 there initiated 5-6 homologous flare/CMEs from the sunspot periphery. However, there are controversies in interpreting the nature of flux appearance in the sunspot periphery. Nitta \& Hudson (2001) considered the fact of this region undergoing a dynamic restructuring due to flux emergence in the form of EFRs. Zhang \& Wang (2002) identified that the main magnetic changes are flux emergence in the form of MMFs. A same type but less active sunspot was seen in NOAA AR8375, for which the magnetic evolution and activity were described by Yurchyshyn \& Wang (2001), Zhang, Solanki \& Wang (2003), and Yang et al. (2004). In this contribution we focus on the flux appearance in AR 9236, and to address: 1) what types of flux appearance can be seen in the sunspot periphery, 2) are they distinct from flux appearance in other environment, 3) how do they associate with flare/CMEs initiated from in the AR?

\section{Two types of flux appearance and CME initiation}

We identified two typical forms of flux appearance in AR9236. They are MMFs and EFRs. Figure 1 shows MDI magnetograms in time sequence. Unlike in AR8375, the MMFs in the sunspot moat did not show much in pairs of opposite polarities. The average magnetic flux of EFRs and MMFs is $10^{19-20}$ and $10^{18} \mathrm{Mx}$ respectively. Their horizontal velocity is about $0.4 \mathrm{~km} / \mathrm{s}$ although the MMFs were always showing a bit faster. EFRs in the sunspot periphery are peculiar in the sense that grow and separate along the circle encircling the sunspot, their magnetic orientation is always the same. For AR9236, the negative flux rotated clockwise, while the positive flux, anti-clockwise. They emerged successively and interact one another, making the magnetic configuration

$\dagger$ Present address: A20 Datun Rd., Chaoyang Dist., Beijing 100012, China 


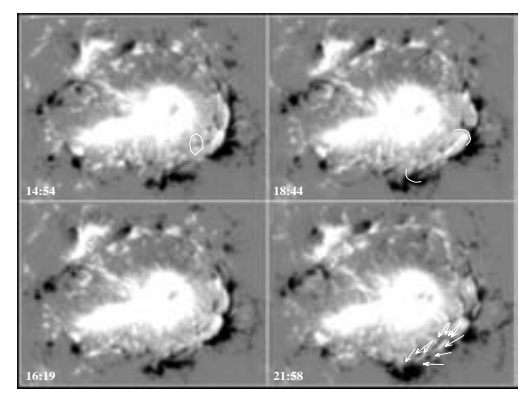

Figure 1. Time sequence of MDI magnetograms of AR9236 on Nov.24 2000. Two pairs of MMFs and one peculiar EFR are marked by polygon and brackets, respectively. Arrows indicate many folds of the magnetic neutral lines which is created by interacting EFRs.

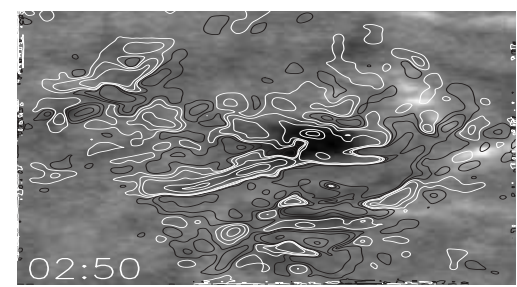

Figure 2. $H_{\beta}$ filtergram of AR9236 taken in the earliest phase of flare/CME at 02:50/03:30 UT on November 26, 2000. Superposed are contours of current helicity. Darker (light) contours indicate the negative (positive helicity).

very complicated. Often there appear multi-fold magnetic neutral lines (see arrows in Figure 1). To understand the CME initiation in such magnetic environments, we select the flare/CME at 02:50/03:30 UT of Nov.26 shown in Figure 2 for this study. The earliest flare patches were closely correlated with the interaction of EFRs, which created multifold magnetic neutral lines and strong magnetic shear. Such structures are much easier to place a premium on the flare/CMEs. For this flare/CME event, EFRs are, indeed, more responsible. It can not be excluded that MMFs also play some role in the magnetic evolution leading to the flare/CME. As revealed by Wang et al. (2004), the flare/CME initiation site is characterized by the close contacting and canceling of magnetic flux of opposite helicities EFRs identified in AR8375 and AR9236 have the same helicity sign of the sunspot, predominately; while the MMFs have opposite helicity sign to the sunspot usually. MMFs brought up the opposite helicity to the AR, which may help with triggering the opening of the overall fields. Other flare/CMEs in AR9236 seem to share the common magnetic configuration and evolution.

\section{Acknowledgements}

This work is supported by National Natural Science Foundation of China (10233050) and National Key Basic Research Foundation of China (G2000078404).

\section{References}

Nitta, N. V. \& Hudson, H. S. 2001, GRL 28, 3801

Yang, X. L., Song, W. B., Zhou, G. P., Zhang, J. \& Wang, J. X. 2004, ASR submitted

Yurchyshyn, V. B. \& Wang, H. M. 2001, Solar Phys. 202, 309

Zhang, J., Solanki, S. K. \& Wang, J. X. 2003, A\&\& 399, 755

Zhang, J. \& Wang, J. X. 2002, ApJ 566, L117

Wang, J., Zhou, G. P. \& Zhang, J. 2004, ApJ 615, 1021 